\title{
Hyperbolic Cauchy problem and Leray's residue formula
}

\author{
by Susumu Tanabé (Moscow)
}

Pamięci Bogdana Ziemiana

\begin{abstract}
We give an algebraic description of (wave) fronts that appear in strictly hyperbolic Cauchy problems. A concrete form of a defining function of the wave front issued from the initial algebraic variety is obtained with the aid of Gauss-Manin systems satisfied by Leray's residues.
\end{abstract}

0. Introduction. In one of his last works [20], Prof. Bogdan Ziemian pursued the possibility of expressing fundamental solutions to PDEs with the aid of Leray residues. He used this technique to write down the Mellin transform of fundamental solutions to Fuchsian type PDEs and proved that these solutions belong to the class of so-called generalized analytic functions (GAFs).

In this note, we show that the advantage of using Leray's residue formula in hyperbolic Cauchy problems is that it facilitates the calculation of the representative integrals (i.e. a basis of a certain cohomology group) whose summation gives fundamental solutions to the Cauchy problem.

Let us explain in short how Leray's residue formula can be applied to the construction of fundamental solutions. Let $V_{x}=\left\{\xi \in \mathbb{C}^{n}: F\left(\xi, x^{\prime}\right)+x_{0}=0\right\}$ be a complex variety of dimension $n-1$ depending on $x=\left(x^{\prime}, x_{0}\right) \in \mathbb{R}^{m}$ defined by a polynomial $F\left(\xi, x^{\prime}\right)+x_{0}$. Choose a continuous family of cycles $\gamma_{x} \in H_{n-1}\left(V_{x}\right)$. For $x$ "in generic position" the variety $V_{x}$ is smooth and $\gamma_{x}$ depends continuously on $x$. Suppose that $a(\xi)$ is a smooth function defined on $\mathbb{C}^{n}$. In this situation the following equality is called Leray's residue

2000 Mathematics Subject Classification: Primary 35L30, 58K99, 34M35; Secondary 32S40, 78A05.

Key words and phrases: hyperbolic Cauchy problem, Leray's residue formula, GaussManin connexion.

Partially supported by Max Planck Institut für Mathematik, Bonn. 
formula:

$$
I_{a}(x)=\int_{\gamma_{x}} a(\xi) \frac{d \xi}{d F}=\frac{1}{2 \pi i} \int_{\partial \gamma_{x}} \frac{a(\xi)}{F\left(\xi, x^{\prime}\right)+x_{0}} d \xi,
$$

where $\partial \gamma_{x} \in H_{n}\left(\mathbb{C}^{n} \backslash V_{x}\right)$ is the so-called Leray coboundary of the cycle $\gamma_{x}$ which is homologically equivalent to a $S^{1}$-bundle over $\gamma_{x}$. See [14], [19]. If $a(\xi) d \xi=d \psi \wedge d F$ for some $\psi \in \Omega_{\mathbb{C}^{n}}^{n-2}$, then the integral $I_{a}(x)$ defined above must be constantly zero:

$$
I_{a}(x)=\int_{\gamma_{x}} \frac{d \psi \wedge d F}{d F}=\int_{\gamma_{x}} d \psi=0,
$$

which one can see by the Stokes theorem.

Evidently

$$
\int_{\gamma_{x}} F\left(\xi, x^{\prime}\right) a(\xi) \frac{d \xi}{d F}=-x_{0} \int_{\gamma_{x}} a(\xi) \frac{d \xi}{d F} .
$$

From (0.2) and (0.3), we conclude that the important forms $a(\xi) d \xi$ that will give nonzero $I_{a}(x)$ not expressed by other Leray residues must be in the space

$$
{ }^{\prime \prime} H=\frac{\Omega_{\mathbb{C}^{n}}^{n}}{d F \wedge d \Omega_{\mathbb{C}^{n}}^{n-2}+F \Omega_{\mathbb{C}^{n}}^{n}} .
$$

Furthermore (0.1) yields the relation

$$
\int_{\gamma_{x}} \frac{d \omega}{d F}=\frac{d}{d x_{0}} \int_{\gamma_{x}} \omega
$$

for $\omega \in \Omega_{\mathbb{C}^{n}}^{n-1}$. That is to say, the differential equation satisfied by $I_{a}(x)$ does not depend on the choice of a cycle along which one defines the integral.

In his famous work [3], E. Brieskorn has shown that for $F\left(\xi, x^{\prime}\right)$ whose singular fibre $V_{0}$ defines an isolated hypersurface singularity, the space " $H$ is a vector space of finite dimension $\mu$ that coincides with the Milnor number of the singularity $V_{0}=\left\{\xi \in \mathbb{C}^{n}: F(\xi, 0)=0\right\}$. Thus if a fundamental solution $I_{a}(x)$ is expressed by a sum of integrals of forms $b_{1} d \xi, \ldots, b_{\mu} d \xi \in{ }^{\prime \prime} H$, for certain $F\left(\xi, x^{\prime}\right)$ we can expect that analytic properties of the fundamental solution can be deduced from those of $I_{b_{1}}(x), \ldots, I_{b_{\mu}}(x)$ which in their turn can be described by information on the singularity $V_{0}$.

To pursue further this study, we propose to make use of the Gauss-Manin system associated with the fibre bundle structure that naturally arises in integration. Our main tool is concrete expressions of overdetermined differential systems obtained from nontrivial relations between base elements of " $H$ for isolated complete intersection singularities (Proposition 5, Theorem 7). In our situation, Leray's residue formula can be written down like (2.10) 
below. The main Theorem 10 directly follows from Theorem 7 on reducing the situation to a specific mapping (2.15).

In our former work [18] we illustrated in concrete examples the possibility of interpreting the fundamental solution to the Cauchy problem associated with the wave operator

$$
P\left(D_{t}, D_{x}\right)=\left(\frac{\partial}{\partial t}\right)^{2}-\sum_{j=1}^{n}\left(\frac{\partial}{\partial x_{j}}\right)^{2}
$$

as a generalized hypergeometric function. In this note we consider the Cauchy problem associated with a general strictly hyperbolic operator with constant coefficients. The procedure to establish a system of differential equations as Leray's residues has been realized with the aid of the general theory of the Gauss-Manin systems for isolated complete intersection singularities [5]. More systematic explanation of this situation from singularity theoretical point of view is given in [1] and [16]-[18].

1. Preliminaries on the Cauchy problem. In this section we prepare fundamental notations and lemmata to be used in further sections. Let $P\left(D_{t}, D_{x}\right)$ be a strictly hyperbolic operator of degree $m$ with constant coefficients, i.e. its total symbol

$$
P(\tau, \xi)=\tau^{m}+\sum_{i=1}^{m} P_{m-i}(\xi) \tau^{m-i}, \quad P_{m-i}(\xi)=\sum_{|\alpha|=i} P_{m-i, \alpha} \xi^{\alpha} \in \mathbb{R}[\xi]
$$

has the decomposition

$$
P(\tau, \xi)=\prod_{j=1}^{m}\left(\tau-\lambda_{j}(\xi)\right)
$$

such that $\lambda_{j}(\xi) \in \mathbb{R}$ if $\xi \in \mathbb{R}^{n}$ and $\lambda_{i}(\xi) \neq \lambda_{j}(\xi)$ for $i \neq j, \xi \in \mathbb{R}^{n} \backslash\{0\}$.

Without loss of generality, we suppose that $P(\tau, \xi)$ is an irreducible polynomial in $\mathbb{R}[\tau, \xi]$. If $P(\tau, \xi)=P_{1}(\tau, \xi) \cdot P_{2}(\tau, \xi)$ the fundamental solution of $P\left(D_{t}, D_{x}\right)$ is a sum of those of $P_{1}\left(D_{t}, D_{x}\right)$ and $P_{2}\left(D_{t}, D_{x}\right)$ provided that their characteristic roots are mutually distinct out of the origin. Let us consider the following Cauchy problem (C.P.):

$$
\left\{\begin{array}{l}
P\left(D_{t}, D_{x}\right) u(t, x)=0, \\
D_{t}^{m-1} u(0, x)=v(x), \\
D_{t}^{m-j} u(0, x)=0, \quad 2 \leq j \leq m,
\end{array}\right.
$$

where $t \in \mathbb{R}, x \in \mathbb{R}^{n}, D_{t}=\frac{\partial}{i \partial t}, D_{x}=\left(\frac{\partial}{i \partial x_{1}}, \ldots, \frac{\partial}{i \partial x_{n}}\right), i=\sqrt{-1}$. We will study the Cauchy problem (C.P.) under the following conditions (C.1), (C.2), (C.3) imposed on the initial data. In order to describe these conditions, we write $\chi_{q}^{\varepsilon}(z)(\varepsilon= \pm 1)$ for the following distributions defined as boundary values of an analytic function on $\mathbb{C}_{z}^{1} \backslash\{0\}$ (cf. [4]): 


$$
\chi_{q}^{\varepsilon}(z)=\chi_{q}(z+i 0)+\varepsilon \chi_{q}(z-i 0),
$$

where

$$
\chi_{q}(z)= \begin{cases}\Gamma(-q) z^{q}, & q \notin \mathbb{Z} \text { or } q \text { a negative integer, } \\ \frac{z^{q}}{q !}\left(-\log z+C_{q}\right), & q \text { a positive integer, }\end{cases}
$$

where $C_{0}=0, C_{q}=C_{q-1}+1 / q$. Note that

$$
\frac{d}{d z} \chi_{q}(z)=\chi_{q-1}(z) \text {. }
$$

(C.1) The initial data are given by a distribution of finite order with singular support (see Definition 2.2.3 of [9]) located on the cotangent bundle of a smooth algebraic surface $S:=\left\{x \in \mathbb{R}^{n}: F(x)-s=0\right\}$ defined by a real polynomial $F(x)$,

$$
v(x)=g(x) \chi_{q}^{\varepsilon}(F(x)-s)
$$

with a smooth function $g(x)$.

We denote the singular support of a distribution $v(x)$ by S.S. $v(x)$.

We also impose several technical conditions in order that a reasoning on isolated complete intersection singularities can be applied to our (C.P.)

(C.2) (Quasihomogeneity) There exist positive integers $w_{1}, \ldots, w_{n}$ such that: (1) $w_{i} \neq w_{j}$ for some $1 \leq i \neq j \leq n,(2)$ G.C.D. $\left(w_{1}, \ldots, w_{n}\right)=1$, and (3) for a positive integer $w(F)$,

$$
\left(\sum_{1 \leq j \leq n} w_{j} x_{j} \frac{\partial}{\partial x_{j}}\right) F(x)=w(F) F(x) .
$$
below.

The above condition (1) plays an essential rôle in establishing Lemma 8 (C.3) $\quad \mathbb{R}[x] / \mathcal{I}$ is a vector space of finite dimension, where

$$
\mathcal{I}=\left\langle F(x), \frac{\partial F}{\partial x_{1}}(x), \ldots, \frac{\partial F}{\partial x_{n}}(x)\right\rangle
$$

(the ideal generated by the entries).

Let us introduce the following notations.

(a) The phase function $\psi(x, t, z)$ is defined as follows:

$$
\psi(x, t, z)=P\left(\left\langle x-z, \operatorname{grad}_{z} F(z)\right\rangle, t \operatorname{grad}_{z} F(z)\right) .
$$

(b) The paired oscillatory integrals studied in [4] are defined for the phase function $\psi(x, t, z)$ introduced in (a):

$$
I_{p}^{\varepsilon}(x, t, s)=\int_{\{F(z)=s\}} H_{p}(z) \chi_{p}^{\varepsilon}(\psi(x, t, z)) \frac{d z}{d F},
$$


with regular amplitude functions of a pseudo-differential operator,

$$
H_{p}(z) \sim \sum_{r=p-m}^{-\infty} h_{p, r}(z) \in S^{p-m}\left(\mathbb{R}^{n}\right),
$$

in which $h_{p, r}(z)$ is homogeneous of order $r$ for large values of $z$. One should understand $I_{p}^{\varepsilon}(x, t, s)$ as a Gel'fand-Leray integral (see 1.5 of [3]) defined on the real algebraic set $S=\left\{z \in \mathbb{R}^{n}: F(z)=s\right\}$.

(c) $\phi(x, t, s)$ is a defining function of the (wave) front $\Sigma$ issued from $S$ determined by (C.P.).

Proposition 1. With the notations introduced in (a)-(c) as above, the following assertion holds:

(d) The solution $u(x, t)$ to the Cauchy problem (C.P.) admits an asymptotic expansion

$$
u(x, t) \sim \sum_{j=0}^{\infty} I_{-n / 2+q+j}^{\varepsilon}(x, t, s) .
$$

That is to say, for every $N \gg 0$ there exists $C_{N}>0$ such that

$$
\left|u(x, t)-\sum_{j=0}^{N} I_{-n / 2+q+j}^{\varepsilon}(x, t, s)\right| \leq C_{N}|\phi(x, t, s)|^{q+N+1},
$$

in the neighbourhood of S.S.u(x,t).

Proof. We give only a sketch of proof; the details with appear in [17], [18]. First of all we show that the phase function of the integrals $I_{p}^{\varepsilon}(x, t, s)$ in (a) is given by (c). Solving the Hamilton-Jacobi equation associated with the Hamiltonian $\tau-\lambda_{\kappa}(\xi), 1 \leq \kappa \leq m$ (in symplectic coordinates with canonical symplectic form $d t \wedge d \tau+\sum_{j=1}^{n} d x_{j} \wedge d \xi_{j}$; for symplectic geometry see Chapter XXI of [9]),

$$
\left\{\begin{array}{l}
\dot{t}=1 \\
\dot{\tau}=0 \\
\dot{x}_{i}=\partial \lambda_{\kappa}(\xi) / \partial \xi_{i} \\
\dot{\xi}_{i}=0, \\
x_{i}(0)=z_{i}, \quad 1 \leq i \leq n, F(z)=s
\end{array}\right.
$$

we get

$$
x_{i}=t \frac{\partial \lambda_{\kappa}(\xi)}{\partial \xi_{i}}+z_{i} \quad \text { with } z \in S=\left\{z \in \mathbb{R}^{n}: F(z)=s\right\} .
$$

This means that the singularities of the solutions to (C.P.) lie on the rays (1.3). These lines are interpreted as rays issued from the initial front $S$ in 
directions determined by the Hamiltonian

$$
P(\tau, \xi)=\tau^{m}+\sum_{i=1}^{m} P_{m-i}(\xi) \tau^{m-i}=\prod_{j=1}^{m}\left(\tau-\lambda_{j}(\xi)\right)
$$

in question.

Consequently they are expressed by integrals with phase

$$
\begin{aligned}
\psi(x, t, z) & =\prod_{j=1}^{m}\left(\left\langle x-z, \operatorname{grad}_{z} F(z)\right\rangle-t \lambda_{j}\left(\operatorname{grad}_{z} F(z)\right)\right) \\
& =P\left(\left\langle x-z, \operatorname{grad}_{z} F(z)\right\rangle, t \operatorname{grad}_{z} F(z)\right) .
\end{aligned}
$$

This is the "minimal" algebraic equation describing the wave front in view of the irreducibility of the polynomial $P(\tau, \xi)$. Here we remark that for every $p \in \mathbb{Q}$ and $H(z) \in \mathcal{D}^{\prime}\left(\mathbb{R}_{z}^{n}\right)$, we have

$$
P\left(D_{t}, D_{x}\right) \int_{S} H(z)(\psi(x, t, z))^{p} \frac{d z}{d F}=0 .
$$

One can prove this equality with the aid of Gauss-Stokes' theorem. Thus the question is how to find a series of integrals

$$
I_{p}^{\varepsilon}(x, t, s)=\int_{S} H_{p}(z) \chi_{p}^{\varepsilon}(\psi(x, t, z)) \frac{d z}{d F}, \quad p \in \mathbb{Q},
$$

whose sum, suitably converging, produces a distribution $u(x, t)$ satisfying (C.1). The possibility of an asymptotic expansion (1.2) consisting of terms like (b) can be proven by well known stationary phase estimates ([9], Theorem 7.7.12). More precisely, let us recall the following lemma.

Lemma 2. Let $(G)^{0}(x, t, s)$ be the residue of a smooth function $G(x, t, z)$ after division by the Jacobi ideal generated by $\partial \psi(x, t, z) / \partial z_{j}, \partial F(z) / \partial z_{j}$, $1 \leq j \leq n$, and $F(z)-s$, i.e.

$$
\begin{aligned}
G(x, t, z)= & G^{0}(x, t, s)+\sum_{j=1}^{n} f_{j}(x, t, s, z) \frac{\partial \psi(x, t, z)}{\partial z_{j}} \\
& +\sum_{j=1}^{n} g_{j}(x, t, s, z) \frac{\partial F(z)}{\partial z_{j}}+h(x, t, s, z)(F(z)-s),
\end{aligned}
$$

with some smooth functions $h(x, t, s, z), f_{j}(x, t, s, z), g_{j}(x, t, s, z), 1 \leq j \leq n$. Then for every smooth function $a(z)$ the following asymptotic estimate with some $C_{N}>0$ holds in the neighbourhood of the wave front $\Sigma=\{(x, t) \in$ $\left.\mathbb{R}^{n+1}:(\psi)^{0}(x, t, s)=0\right\}:$ 


$$
\begin{array}{r}
\left|\int_{S} a(z) \chi_{q}^{\varepsilon}(\psi(x, t, z)) d z-\sum_{j=0}^{N}\left(L_{\psi, j} a\right)^{0}(x, t, s) \chi_{q+n / 2+j}^{\varepsilon}\left((\psi)^{0}(x, t, s)\right)\right| \\
<C_{N}\left|(\psi)^{0}(x, t, s)\right|^{n / 2+N+1+q}
\end{array}
$$

with differential operators $L_{\psi, j}$ of degree $2 j$. Furthermore we have

$$
\left(L_{\psi, 0} a\right)^{0}(x, t, s)=i^{n / 2}(2 \pi)^{(n-1) / 2}(a)^{0}(x, t, s)\left|\operatorname{det}\left(\frac{\psi_{z z}}{2 \pi i}\right)^{0}(x, t, s)\right|^{-1 / 2}
$$

In the literature concerning singularity theory, one often calls the correspondence $G(x, t, z) \mapsto G^{0}(x, t, s)$ the Lyashko-Loojenga mapping.

Let us briefly sketch the proof of the lemma. Malgrange's division theorem yields the decomposition (1.6) in connexion with the fact that the follow-

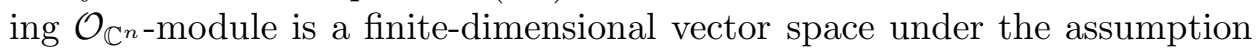
(C.3):

$$
\frac{\Omega_{\mathbb{C}^{n}}^{n}}{d F(z) \wedge \Omega_{\mathbb{C}^{n}}^{n-1}+d \psi(0,0, z) \wedge \Omega_{\mathbb{C}^{n}}^{n-1}+d_{z} \Omega_{\mathbb{C}^{n}}^{n-1}+F(z) \wedge \Omega_{\mathbb{C}^{n}}^{n}} .
$$

Further it suffices to apply the above mentioned stationary phase method.

By Lemma 2 the function $(\psi)^{0}(x, t, s)$ can be given by $(1.5)$ for $G(x, t, z)$ $=\psi(x, t, z)$ in (1.4). As a defining function $\phi(x, t, s)$ of the wave front issued from $S$, one can take a polynomial $\phi$ such that $\left\{(x, t) \in \mathbb{R}^{n+1}:(\psi)^{0}(x, t, s)\right.$ $=0\} \subset\left\{(x, t) \in \mathbb{R}^{n+1}: \phi(x, t, s)=0\right\}$ and $S=\{x: \phi(x, 0, s)=0\}$. This $\phi(x, t, s)$ is as needed in (c).

It remains to justify the asymptotic estimates in (b) and (d). This can be achieved in view of (1.6) and a well known construction of an elementary solution to the strictly hyperbolic Cauchy problem (see for example [7], [8]). In particular, due to the choice of $\phi(x, t, s)$, the inequality (1.2), satisfied for $\psi^{0}(x, t, s)$ in a local context, holds for $\phi(x, t, s)$. The assertion follows.

We formulate a simple lemma before introducing necessary notations.

LEMmA 3. Under the assumptions (C.2), (C.3) imposed on $F(x)$ there exists a collection of polynomials of degree $\leq m, W_{1}(x, t), \ldots, W_{\mu^{\prime}}(x, t)$, with $\mu^{\prime}$ an integer smaller than $m^{n} \prod_{i=1}^{n} w(F) / w_{i}$, satisfying

$$
\left.\psi(x, t, z)=\left\langle z, \operatorname{grad}_{z} F(z)\right)\right\rangle^{m}+\sum_{i=1}^{\mu^{\prime}} W_{i}(x, t) z^{\alpha^{(i)}}
$$

for $\psi(x, t, z)$ of $(1.4)$. Here $\alpha^{(i)}=\left(\alpha_{1}^{(i)}, \ldots, \alpha_{n}^{(i)}\right) \in\left(\mathbb{Z}_{\geq 0}\right)^{n}$ is a multi-index with $\sum_{j=1}^{n} w_{j} \alpha_{j}^{(i)}<m \cdot w(F)$. 
The proof is a direct calculation using (1.4). Quasihomogeneity of $F(z)$ yields the estimate on $\mu^{\prime}$. Let us denote by

$$
w\left(z^{\alpha^{(i)}}\right)=\sum_{j=1}^{n} w_{j} \alpha_{j}^{(i)}
$$

the quasihomogeneous weight of the monomial $z^{\alpha^{(i)}}$ for $\alpha^{(i)} \in \mathbb{N}^{n}$. In terms of this weight we distinguish two cases.

CASE 1. If there is a term with $w\left(z^{\alpha^{(i)}}\right)=0$ in (1.7), let us mark it as $\alpha^{(1)}$ and define a polynomial

$$
\left.f_{1}(y(x, t), z)=\left\langle z, \operatorname{grad}_{z} F(z)\right)\right\rangle^{m}+\sum_{i=1}^{\mu^{\prime}} y_{i}(x, t) z^{\alpha^{(i)}} .
$$

Here $y_{i}(x, t)=W_{i}(x, t), 1 \leq i \leq \mu^{\prime}$, are the polynomials introduced in Lemma 3.

CASE 2. If all terms of (1.7) have positive weight, we define

$$
\left.f_{1}(y(x, t), z)=\left\langle z, \operatorname{grad}_{z} F(z)\right)\right\rangle^{m}+\sum_{i=2}^{\mu^{\prime}+1} y_{i}(x, t) z^{\alpha^{(i-1)}}+y_{1}
$$

with $y_{i+1}(x, t)=W_{i}(x, t), 1 \leq i \leq \mu^{\prime}$.

For simplicity we set $\mu=\mu^{\prime}$ for Case 1 and $\mu=\mu^{\prime}+1$ for Case 2 .

Further we define

$$
I_{p}(y(x, t), s)=\int_{S} H_{p}(z) \chi_{p}\left(f_{1}(y(x, t), z)\right) \frac{d z}{d F} .
$$

Hence if one writes $y^{\prime}=\left(y_{2}(x, t), \ldots, y_{\mu}(x, t)\right)$, then

$$
I_{p}^{\varepsilon}(x, t, s)=I_{p}\left(y_{1}+i 0, y^{\prime}(x, t), s\right)+\varepsilon I_{p}\left(y_{1}-i 0, y^{\prime}(x, t), s\right)
$$

on the understanding that the boundary value is taken at $y_{1}=0$ in Case 2 . Thus it is essential to study $I_{p}(y(x, t), s)$ to estimate the asymptotic behaviour of $I_{p}^{\varepsilon}(x, t, s)$. From now on we shall regard the integral (1.8) as a function of the variables $y(x, t)=\left(y_{1}, y_{2}(x, t), \ldots, y_{\mu}(x, t)\right)$. Therefore our main concern will be to investigate the differential equations satisfied by $I_{p}(y, s)$ corresponding to various amplitudes $H_{p}(z)$ with the aid of the Gauss-Manin connexions associated with complete intersection singularities.

2. Gauss-Manin connexions for quasihomogeneous complete intersections. We propose to study the integrals $I_{p}(y, s)$ defined in (1.8) by means of the Gauss-Manin system associated with complete intersection singularities. It is well known that the Gauss-Manin connexion can be defined 
on the relative de Rham cohomology groups. Here we propose to calculate it on so-called Brieskorn lattices (see [3], [5]).

The formulation of this section is a modification of [16], $\S 1$, adapted to our situation.

Consider a mapping $f: X \rightarrow Y$ between complex manifolds $X=$ $\left(\mathbb{C}^{N+K}, 0\right)$ and $Y=\left(\mathbb{C}^{K}, 0\right)$ that defines an isolated quasihomogeneous complete intersection singularity at the origin. That is to say, if we set

$$
X_{y}:=\left\{u \in X: f_{0}(u)=y_{0}, \ldots, f_{K-1}(u)=y_{K-1}\right\},
$$

then $\operatorname{dim} X_{y}=N \geq 0$ and the critical set of the mapping $f: X_{0} \rightarrow Y$ is isolated in $X_{0}$. Further we assume that the polynomials $f_{0}(u), \ldots, f_{K-1}(u)$ are quasihomogeneous, i.e. there exists a collection of positive integers $v_{1}, \ldots$ $\ldots, v_{N+K}$ whose greatest common divisor equals 1 and

$$
\left(v_{1} u_{1} \frac{\partial}{\partial u_{1}}+\ldots+v_{N+K} u_{N+K} \frac{\partial}{\partial u_{N+K}}\right) f_{l}(u)=p_{l} f_{l}(u), \quad l=0,1, \ldots, K-1,
$$

for certain integers $p_{0}, \ldots, p_{K-1}$. We shall call the vector field

$$
E=\sum_{i=1}^{N+K} v_{i} u_{i} \frac{\partial}{\partial u_{i}}
$$

the Euler vector field and $v_{1}, \ldots, v_{N+K}$ (resp. $p_{0}, \ldots, p_{K-1}$ ) the positive weights of the variables $u_{1}, \ldots, u_{N+K}$ (resp. polynomials $f_{0}, \ldots, f_{K-1}$ ), i.e. $v_{1}=w\left(u_{1}\right), p_{0}=w\left(f_{0}\right)$ etc.

In order to calculate the Gauss-Manin connexion for the isolated complete intersection singularity $X_{0}$, we introduce two vector spaces $V$ and $F$. Following Greuel-Hamm [6], we look at a space whose dimension as vector space over $\mathbb{C}$ is known to be the Milnor number $\mu\left(X_{0}\right)$,

$$
\begin{aligned}
& V:= \\
& \frac{\Omega_{X}^{N}}{d f_{0} \wedge \Omega_{X}^{N-1}+\ldots+d f_{K-1} \wedge \Omega_{X}^{N-1}+d \Omega_{X}^{N-1}+f_{0} \Omega_{X}^{N}+\ldots+f_{K-1} \Omega_{X}^{N}} .
\end{aligned}
$$

The second one will later turn out to be isomorphic to $V$ (see Proposition 6):

$$
F:=\frac{\Omega_{X}^{N+1}}{d f_{0} \wedge \Omega_{X}^{N}+\ldots+d f_{K-1} \wedge \Omega_{X}^{N}+i_{E}\left(\Omega_{X}^{N+2}\right)} .
$$

Here $i_{E}$ means the inner contraction with the Euler field $E$ defined by (2.2). The third vector space associated with the singularity $X_{0}$ is defined as follows:

$$
\Phi:=\frac{\Omega_{X}^{N+K}}{d f_{0} \wedge \ldots \wedge d f_{K-1} \wedge \Omega_{X}^{N}+f_{0} \Omega_{X}^{N+K}+\ldots+f_{K-1} \Omega_{X}^{N+K}} .
$$

Later we define period integrals as coupling of forms of $V$ or of $\Phi$ with basic elements of the homology groups $H_{N}\left(X_{y}\right)$. We also recall the definition of 
the Brieskorn lattice " $H$ from [5]:

$$
{ }^{\prime \prime} H=\frac{\Omega_{X}^{N+K}}{d f_{0} \wedge \ldots \wedge d f_{K-1} \wedge d \Omega_{X}^{N-1}},
$$

whose rank as $\mathcal{O}_{Y}$-module equals the Milnor number $\mu\left(X_{0}\right)$. It is easy to show

Lemma 4. For quasihomogeneous polynomials $f_{0}, \ldots, f_{K-1}$ defining an isolated complete intersection singularity,

$$
\Phi \cong{ }^{\prime \prime} H /\left(f_{0}, \ldots, f_{K-1}\right) .
$$

Thus $\operatorname{dim}_{\mathbb{C}} \Phi=\mu\left(X_{0}\right)$.

Let us fix a set of quasihomogeneous $N+1$-forms $\widetilde{\omega}_{1}, \ldots, \widetilde{\omega}_{\mu\left(X_{0}\right)}$ whose residue classes form a base of $F$. Similarly we fix a set of $N+K$ quasihomogeneous forms $\phi_{1}(u) d u, \ldots, \phi_{\mu\left(X_{0}\right)}(u) d u$ whose residue classes give a base of $\Phi$.

From definitions (2.4) and (2.5) we easily deduce the following.

Proposition 5. For each form $\widetilde{\omega}_{i}$, one has the following decomposition:

$$
\begin{aligned}
\widetilde{\omega}_{i} \wedge d f_{0} \wedge & \stackrel{l}{\vee} . \wedge d f_{K-1} \\
& =\sum_{j=1}^{\mu\left(X_{0}\right)} P_{i j}^{(l)} \phi_{j}(u) d u \bmod \left(d f_{0} \wedge \ldots \wedge d f_{K-1} \wedge d \Omega_{X}^{N-1}\right)
\end{aligned}
$$

with $P_{i j}^{(l)} \in \mathbb{C}\left[f_{0}, \ldots, f_{K-1}\right]$ and $\phi_{j}(u) d u$ as above for $1 \leq i, j \leq \mu\left(X_{0}\right)$, $0 \leq l \leq K-1$ and $d f_{1} \wedge \stackrel{l}{\stackrel{l}{.}}$. $\wedge d f_{K-1}=\bigwedge_{i \neq l} d f_{i}$.

As a matter of fact the right-hand side of (2.6) can be considered as an element of ${ }^{\prime \prime} H$. From $[16]$ we recall the following

Proposition 6. Under the situation and definitions as above, the mapping $i_{E}: F \rightarrow V$ induces an isomorphism. Consequently, $\operatorname{dim}_{\mathbb{C}} F=\operatorname{dim}_{\mathbb{C}} V$ $=\mu\left(X_{0}\right)$.

In view of Proposition 6, let us choose a base $\widetilde{\omega}_{i}$ of $F$ (resp. $\omega_{i}$ of $V$ ) such that $\widetilde{\omega}_{i}=\left(1 / l_{i}\right) d \omega_{i}$ where $l_{j}$ denotes the weight of the form $\omega_{j}$. Note that $i_{E} \widetilde{\omega}_{i} \equiv\left(1 / l_{i}\right)\left(d i_{E}+i_{E} d\right)\left(\omega_{i}\right) \equiv \omega_{i}, 1 \leq i \leq \mu\left(X_{0}\right)$, in $F$. To make a transition from $(N+K)$-forms to period integrals, we introduce meromorphic $N$-forms $\psi_{i}$ satisfying

$$
d f_{0} \wedge \ldots \wedge d f_{K-1} \wedge \psi_{i}=\phi_{i}(u) d u, \quad 1 \leq i \leq \mu\left(X_{0}\right) .
$$


Then we derive the following relation from Proposition 5:

$$
\begin{aligned}
& d \omega_{j}=l_{j} \widetilde{\omega}_{j} \equiv l_{j}\left(\sum_{q=1}^{\mu\left(X_{0}\right)} P_{j q}^{(0)} d f_{0} \wedge \psi_{q}+\ldots\right. \\
& \left.+(-1)^{K-1} \sum_{q=1}^{\mu\left(X_{0}\right)} P_{j q}^{(K-1)} d f_{K-1} \wedge \psi_{q}\right) \bmod \left(\left(d f_{0}, \ldots, d f_{K-1}\right) d \Omega_{X}^{N-1}\right) .
\end{aligned}
$$

See (2.12) below to see that this relation calculates the "partial derivative" $\frac{d \omega}{d f_{i}}$. Hence,

$$
\begin{array}{r}
\omega_{j} \equiv i_{E}\left(\widetilde{\omega}_{j}\right) \equiv \sum_{i=0}^{K-1}(-1)^{i}\left[\sum_{q=1}^{\mu\left(X_{0}\right)} P_{j q}^{(i)} p_{i} f_{i} \psi_{q}-\sum_{q=1}^{\mu\left(X_{0}\right)} P_{j q}^{(i)} d f_{i} \wedge i_{E}\left(\psi_{q}\right)\right] \\
\bmod \left(\left(d f_{0}, \ldots, d f_{K-1}\right) i_{E} d \Omega_{X}^{N-1},\left(f_{0}, \ldots, f_{K-1}\right) d \Omega_{X}^{N-1}\right) .
\end{array}
$$

As a consequence,

$$
\begin{aligned}
d \omega_{j} & \equiv \sum_{q=1}^{\mu\left(X_{0}\right)}\left[\sum_{i=0}^{K-1}(-1)^{i}\left(d\left(p_{i} P_{j q}^{(i)} f_{i}\right)-w\left(\psi_{q}\right) P_{j q}^{(i)} d f_{i}\right)\right] \wedge \psi_{q} \\
& +\sum_{q=1}^{\mu\left(X_{0}\right)}\left[\sum_{i=0}^{K-1}(-1)^{i} p_{i} P_{j q}^{(i)} f_{i}\right] \wedge d \psi_{q} \bmod \left(\left(d f_{0}, \ldots, d f_{K-1}\right) d \Omega_{X}^{N-1}\right)
\end{aligned}
$$

where $w\left(\psi_{q}\right)$ is the quasihomogeneous weight of the form $\psi_{q}$. The expression (2.9) can be simplified if one couples it with a vanishing $N$-cycle, say $\gamma(y)$, and gives rise to nontrivial relations between integrals $\int_{\gamma(y)} \psi_{q}$, instead of those between forms. One defines the so-called period integral $I_{\phi_{q}, \gamma(y)}(y)$ taken along a vanishing cycle $\gamma(y)$ without caring for its ambiguity in the homology group $H_{N}\left(X_{y}, \mathbb{Z}\right)$ for the moment:

$$
\begin{aligned}
I_{\phi_{q}, \gamma(y)}(y) & :=\int_{\gamma(y)} \psi_{q}=\left(\frac{1}{2 \pi i}\right)^{K} \int_{\partial \gamma(y)} \frac{d f_{0} \wedge \ldots \wedge d f_{K-1} \wedge \psi_{q}}{\left(f_{0}-y_{0}\right) \ldots\left(f_{K-1}-y_{K-1}\right)} \\
& =\left(\frac{1}{2 \pi i}\right)^{K} \int_{\partial \gamma(y)} \frac{\phi_{q}(u) d u}{\left(f_{0}-y_{0}\right) \ldots\left(f_{K-1}-y_{K-1}\right)},
\end{aligned}
$$

where $\partial \gamma(y) \in H_{N+K}\left(\mathbb{C}^{N+K} \backslash \bigcup_{i=0}^{K-1}\left\{f_{i}=y_{i}\right\}, \mathbb{Z}\right)$ is a cycle obtained with the aid of Leray's coboundary operator $\partial$. That is to say, although $\psi_{q}$ is in general a meromorphic form with poles along the critical set of the mapping $f$, $I_{\phi_{q}, \gamma(y)}(y)$ can be calculated as the integral of a holomorphic form on $\partial \gamma(y)$.

One may consult a booklet by F. Pham [14], or a book by V. A. Vassiliev [19] on the coboundary operator. One views (2.10) as Leray's residue formula in our situation (2.1). 
From (2.8) we can deduce

$$
\int_{\gamma(y)} \omega_{j}=\sum_{q=1}^{\mu\left(X_{0}\right)}\left[\sum_{i=0}^{K-1}(-1)^{i} p_{i} y_{i} P_{j q}^{(i)}(y)\right] I_{\phi_{q}, \gamma(y)}(y) .
$$

This is easily seen from (2.10) and the following evident equalities:

$$
\begin{gathered}
\int_{\partial \gamma(y)} \frac{d f_{0} \wedge \ldots \wedge d f_{K-1}}{\left(f_{0}-y_{0}\right) \ldots\left(f_{K-1}-y_{K-1}\right)} \wedge d f_{i} \wedge i_{E}\left(\psi_{q}\right)=0, \quad 0 \leq i \leq K-1, \\
\int_{\partial \gamma(y)} \frac{d f_{0} \wedge \ldots \wedge d f_{K-1}}{\left(f_{0}-y_{0}\right) \ldots\left(f_{K-1}-y_{K-1}\right)} \wedge d \varphi=0, \quad \varphi \in \Omega_{X}^{N-1} .
\end{gathered}
$$

Let us consider the relation

$$
d \int_{\gamma(y)} \omega_{j}=l_{j} \sum_{q=1}^{\mu\left(X_{0}\right)}\left[\sum_{i=0}^{K-1}(-1)^{i} P_{j q}^{(i)}(y) d y_{i}\right] I_{\phi_{q}, \gamma(y)}(y),
$$

obtained from (2.7) and (2.11). As a result we get equations between $I_{\phi_{q}}(y)$ and $\frac{\partial}{\partial y_{l}} I_{\phi_{q}}, 0 \leq l \leq K-1$ (we do not specify $\gamma(y)$ except in necessary cases):

$$
\begin{aligned}
\frac{\partial}{\partial y_{l}}\left[\sum_{q=1}^{\mu\left(X_{0}\right)} \sum_{i=0}^{K-1}(-1)^{i} p_{i} y_{i} P_{j q}^{(i)} I_{\phi_{q}}\right] \\
\quad=l_{j} \sum_{q=1}^{\mu\left(X_{0}\right)}\left[\sum_{i=0}^{K-1}(-1)^{i} P_{j q}^{(i)}(y) d y_{i}\right] I_{\phi_{q}}(y), \quad 1 \leq j \leq \mu\left(X_{0}\right) .
\end{aligned}
$$

Thus we have obtained a system of differential equations to be understood as the Gauss-Manin connexion of the singularity $X_{0}$. To state the theorem in a simple form, we introduce the following notations:

$$
\begin{aligned}
& \mathbb{I}_{V}=\left(\int_{\gamma(y)} \omega_{1}, \ldots, \int_{\gamma(y)} \omega_{\mu\left(X_{0}\right)}\right), \\
& \mathbb{I}_{\Phi}=\left(I_{\phi_{1}, \gamma(y)}(y), \ldots, I_{\phi_{\mu\left(X_{0}\right)}, \gamma(y)}(y)\right),
\end{aligned}
$$

i.e. vectors of integrals taken along a certain vanishing cycle $\gamma(y)$. We define several other $\mu\left(X_{0}\right) \times \mu\left(X_{0}\right)$ matrices as follows:

$$
L_{V}=\operatorname{diag}\left(l_{1}, \ldots, l_{\mu\left(X_{0}\right)}\right)
$$

with $l_{i}=w\left(\omega_{i}\right), P^{(0)}(y)=\left(P_{j q}^{(0)}(y)\right), \ldots, P^{(K-1)}(y)=\left(P_{j q}^{(K-1)}(y)\right), 1 \leq$ $j, q \leq \mu\left(X_{0}\right)$; the elements of these matrices are defined in (2.6).

Combining the above arguments and the theory due to Greuel [5], we obtain the following.

TheOREM 7. (1) For a quasihomogeneous mapping $f: X \rightarrow Y$ with isolated complete intersection singularities of dimension $N$ like (2.1), the 
Gauss-Manin system satisfied by $\mathbb{I}_{\Phi}$ is as follows:

$$
d\left[\sum_{i=0}^{K-1}(-1)^{i} p_{i} y_{i} P^{(i)}(y) \mathbb{I}_{\Phi}\right]=L_{V}\left[\sum_{i=0}^{K-1}(-1)^{i} P^{(i)}(y) d y_{i}\right] \mathbb{I}_{\Phi} .
$$

(2) The critical value $D$ (singular locus of the system (2.13)) of the deformation $X_{y}$ is given by $D=\{y \in Y: \Delta(y)=0\}$ where

$$
\Delta(y)=\operatorname{det}\left(\sum_{i=0}^{K-1}(-1)^{i} p_{i} y_{i} P^{(i)}(y)\right) .
$$

(3) The system of differential equations (2.13) is a holonomic system.

Let us return to the problem (C.P.) of $\S 1$. Our main concern is to understand the integral (1.8) as a sum of integrals like (2.10) for a certain mapping $f$. To adapt our (C.P.) to the scheme explained before Theorem 7, we consider the mapping $f: X \rightarrow Y$ for $X=\left(\mathbb{C}_{u}^{n+\mu}, 0\right), Y=\left(\mathbb{C}_{y}^{\mu+1}, 0\right)$, defined as follows:

$$
\left\{\begin{aligned}
f_{0}(u) & =F(z)=y_{0} \\
f_{1}(u) & =z_{n+\mu}^{P}+\left\langle z, \operatorname{grad}_{z} F(z)\right\rangle^{m}+\sum_{i=1}^{\mu-1} z_{n+i} z^{\alpha^{(i)}}=y_{1} \\
f_{2}(u) & =z_{n+1}=y_{2} \\
& \vdots \\
f_{i+1}(u) & =z_{n+i}=y_{i+1} \\
& \vdots \\
f_{\mu}(u) & =z_{n+\mu-1}=y_{\mu}
\end{aligned}\right.
$$

with the notation $z=\left(z_{1}, \ldots, z_{n}\right), z^{\prime}=\left(z_{n+1}, \ldots, z_{n+\mu-1}\right), u=\left(z, z^{\prime}, z_{n+\mu}\right)$. Here the power $P$ is an integer that corresponds to the denominator of $q \in \mathbb{Q}$.

Lemma 8. For F( $z$ ) satisfying conditions (C.2), (C.3), the mapping (2.15) defines an isolated quasihomogeneous complete intersection singularity

$$
X_{0}=\left\{u \in X: f_{0}(u)=\ldots=f_{\mu}(u)=0\right\} .
$$

Namely $V=\Omega_{X}^{n+\mu} / W$ is a finite-dimensional vector space, where

$$
\begin{aligned}
W= & f_{0} \Omega_{X}^{n+\mu}+f_{1} \Omega_{X}^{n+\mu}+\sum_{i=1}^{\mu} z_{i+n} \Omega_{X}^{n+\mu}+d F \wedge \Omega_{X}^{n+\mu-1} \\
& +d f_{1} \wedge \Omega_{X}^{n+\mu-1}+\sum_{i=1}^{\mu} d z_{i+n} \wedge \Omega_{X}^{n+\mu-1}
\end{aligned}
$$


Proof. The complete intersection property follows from the fact that the polynomials $F(z)$ and $\left\langle z, \operatorname{grad}_{z} F(z)\right\rangle$ are of the same quasihomogeneous weight but with different coefficients. This is a consequence of (C.2)(1), which supposes that $F(z)$ is not a homogeneous polynomial. The condition (C.3) entails immediately the finite dimensionality of $V$.

To see that the components of $\mathbb{I}_{\Phi}$ defined for the mapping (2.15) give rise to integrals of type (1.8), we prepare the following.

Lemma 9. (1) Define

$$
\Phi\left(z, z^{\prime}\right)=\left.\frac{\Omega_{X}^{n+\mu+1}}{d f_{0} \wedge \ldots \wedge d f_{\mu} \wedge \Omega_{X}^{n}+\sum_{i=0}^{\mu} f_{i} \Omega_{X}^{n+\mu+1}}\right|_{z_{n+\mu}=0} .
$$

Then the following natural isomorphism holds:

$$
\Phi \cong \Phi\left(z, z^{\prime}\right) \otimes\left(\mathbb{C}\left[z_{n+\mu}\right] /\left\langle z_{n+\mu}^{P}\right\rangle\right) .
$$

(2) For $\partial \gamma_{n-2} \in H_{n+\mu-1}\left(\left.\mathbb{C}^{n+\mu-1} \backslash \bigcup_{i=0}^{\mu}\left\{f_{i}=y_{i}\right\}\right|_{z_{n+\mu}=0}, \mathbb{Z}\right)$, the Leray coboundary of a vanishing cycle $\gamma_{n-2} \in H_{n-2}\left(\left.X_{y}\right|_{z_{n+\mu}=0}, \mathbb{Z}\right)$ one can choose a corresponding vanishing cycle $\widetilde{\gamma}_{n-1} \in H_{n-1}\left(X_{y}, \mathbb{Z}\right)$ such that

$$
\begin{aligned}
\int_{\partial \gamma_{n-2}} \phi(z)\left(f_{1}\left(z, y_{2}, \ldots, y_{\mu}, 0\right)-y_{1}\right)^{(r+1) / P-1} \frac{d z}{d F} \\
=\varepsilon\left(\frac{1}{2 \pi i}\right)^{\mu} \int_{\partial \widetilde{\gamma}_{n-1}} \phi(z) z_{n+\mu}^{r} \frac{d u}{\left(f_{0}-u_{0}\right) \ldots\left(f_{\mu}-u_{\mu}\right)},
\end{aligned}
$$

where $\varepsilon \in \mathbb{C}^{\times}$with $\varepsilon^{P}=1$. Furthermore, the cycle $\partial \widetilde{\gamma}_{n-1} \in H_{n+\mu}\left(\mathbb{C}^{n+\mu} \backslash\right.$ $\left.\bigcup_{i=0}^{\mu}\left\{f_{i}=u_{i}\right\}, \mathbb{Z}\right)$ is topologically equivalent to the product of a small circle on the complex $z_{n+\mu}$-plane and $\partial \gamma_{n-2}$.

Proof. The statement (1) is evident. The statement (2) is an integral version of statement (1), which can be shown by means of equality (2.10).

Thus the singular locus of the integral (1.8) can be given by that of

$$
\int_{\widetilde{\gamma}_{n-1}} \phi(z) z_{n+\mu}^{r} \frac{d u}{d f_{0} \wedge \ldots \wedge d f_{\mu}}
$$

with $\widetilde{\gamma}_{n-1} \in H_{n-1}\left(X_{y}, \mathbb{Z}\right)$ after substitution $y_{1}=-W_{1}(x, t)$ (Case 1 after Lemma 3), or $y_{1}=0$ (Case 2 after Lemma 3), $y_{i}=W_{i}(x, t), 2 \leq i \leq \mu$. Recall that we denoted the quasihomogeneous weight of the function $f_{i}$ by $p_{i}, 0 \leq i \leq \mu$. We define matrices $P^{(i)}(y), 2 \leq i \leq \mu$, for the mapping (2.15) according to (2.6) and Theorem 7. Combining Theorem 7 with Lemma 9, we obtain the following. 
THEOREM 10. The defining equation of the wave front (Proposition 1(c)) is given by the following polynomial:

$$
\phi(x, t, s)=\left.\operatorname{det}\left(\sum_{i=0}^{\mu}(-1)^{i} p_{i} y_{i} P^{(i)}(y)\right)\right|_{y_{0}=s, y_{i}=W_{i}(x, t), 2 \leq i \leq \mu} .
$$

Here a restriction should be imposed in accordance with the two cases treated just after Lemma 3: $y_{1}=-W_{1}(x, t)$ in Case 1 and $y_{1}=0$ in Case 2.

\section{References}

[1] A. G. Aleksandrov and S. Tanabé, Gauss-Manin connexions, logarithmic forms and hypergeometric functions, in: Geometry from the Pacific Rim (Singapore, 1994), de Gruyter, 1997, 1-21.

[2] P. Appel et J. Kampé de Fériet, Fonctions hypergéometriques et hypersphériques, Gauthier-Villars, Paris, 1926.

[3] E. Brieskorn, Die Monodromie der isolierten Singularitäten von Hyperflächen, Manuscripta Math. 2 (1970), 103-161.

[4] L. Gårding, Sharp fronts of paired oscillatory integrals, Publ. RIMS Kyoto Univ. 12 suppl. (1977), 53-68.

[5] G.-M. Greuel, Der Gauß-Manin Zusammenhang isolierter Singularitäten von vollständigen Durchschnitten, Math. Ann. 214 (1975), 235-266.

[6] G.-M. Greuel und H. Hamm, Invarianten quasihomogener vollständiger Durchschnitten, Invent. Math. 49 (1978), 67-86.

[7] Y. Hamada, The singularities of the solutions of the Cauchy problem, Publ. RIMS Kyoto Univ. 5 (1969), 21-40.

[8] Y. Hamada, J. Leray et C. Wagschal, Systèmes d'équations aux dérivées partielles à caractéristiques multiples: problème de Cauchy ramifié, hyperbolicité partielle, J. Math. Pures Appl. 55 (1976), 297-352.

[9] L. Hörmander, The Analysis of Linear Partial Differential Operators, Vol. I, Springer, 1984.

[10] E. Leichtnam, Le problème de Cauchy ramifié linéaire pour des données à singularités algébriques, Mem. Soc. Math. France 53 (1993).

[11] Kh. M. Malikov, Overdeterminacy of the differential systems for versal integrals of type A, D, E, Differentsial'nye Uravneniya 18 (1982), 1394-1400 (in Russian).

[12] V. P. Palamodov, Deformations of complex spaces, in: Several Complex Variables IV, Encyclopedia Math. Sci. 10, Springer, 1990, 105-194.

[13] I. G. Petrovskiŭ, On the diffusion of waves and the lacunas for hyperbolic equations, Mat. Sb. 17 (1945), 289-370.

[14] F. Pham, Introduction à l'étude topologique des singularités de Landau, GauthierVillars, 1967.

[15] S. Tanabé, Lagrangian variety and the condition for the presence of sharp front of the fundamental solution to Cauchy problem, Sci. Papers College Arts Sci. Univ. Tokyo, 42 (1992), 149-159.

[16] - Transformée de Mellin des intégrales fibres de courbe espace associées aux singularités isolées d'intersection complète quasihomogènes, Compositio Math., to appear. 
[17] S. Tanabé, Connexion de Gauss-Manin associée à la déformation verselle des singularités isolées d'hypersurface et son application au XVIe problème de Hilbert, preprint.

[18] -, On geometry of fronts in wave propagations, in: Geometry and Topology of Caustics-Caustics'98, Banach Center Publ. 50, Inst. Math., Polish Acad. Sci., 1999, 287-304.

[19] V. A. Vassiliev, Ramified Integrals, Singularities and Lacunas, Kluwer, Dordrecht, 1995.

[20] B. Ziemian, Leray residue formula and asymptotics of solutions to constant coefficient PDEs, Topol. Methods Nonlinear Anal. 3 (1994), 257-293.

Moscow Independent University

Bol'shoŭ Vlasievskiǔ pereulok 11

121002, Moscow, Russia

E-mail: tanabe@mpim-bonn.mpg.de

tanabe@mccme.ru

tanabesusumu@hotmail.com

Reçu par la Rédaction le 1.7.1999

Révisé le 12.9.2000 